\title{
EFFECT OF NANOCRYSTALLIZATION PROCESS ON THE MAGNETOMECHANICAL COUPLING IN $\mathrm{Fe}-\mathrm{Si}-\mathrm{B}-\mathrm{Cu}-\mathrm{Nb}$ ALLOY
}

\author{
Z. KaCZkowski, L. MaLkińSKI, M. KuŹMIŃski \\ Institute of Physics, Polish Academy of Sciences \\ Al. Lotników 32/46, 02-668 Warszawa, Poland \\ AND M. MÜLLER \\ Institute of Solid State and Materials Research \\ Helmholtz Str. 20, O-8020 Dresden, Germany
}

\begin{abstract}
Magnetic and magnetoelastic properties of the $\mathrm{Fe}_{73.5} \mathrm{Si}_{16.5} \mathrm{~B}_{6} \mathrm{Cu}_{1} \mathrm{Nb}_{3}$ alloy change considerably during nanocrystallization process. Improvement of magnetic properties is accompanied by the decrease in piezomagnetic parameters such as magnetomechanical coupling and $\Delta E$-effect.
\end{abstract}

PACS numbers: 75.80.+q; 75.60.Nt, 75.60.Ch

\section{Samples and measuring methods}

Nanocrystalline or "FINEMET" alloys [1] can be produced from some amorphous alloys by the proper annealing treatment. Samples of amorphous alloy of composition $\mathrm{Fe}_{73.5} \mathrm{Si}_{16.5} \mathrm{~B}_{6} \mathrm{Cu}_{1} \mathrm{Nb}_{3}$ were annealed at temperatures ranging from $490^{\circ} \mathrm{C}$ to $600^{\circ} \mathrm{C}$ for 5 minutes in order to produce different degree of nanocrystalline phase in the samples. The heating rate was about $20 \mathrm{~K} / \mathrm{min}$. The annealings were performed at helium atmosphere in the presence of saturating magnetic field, oriented transversely to the sample length. Resonance-antiresonance method [2] was used to measure magnetomechanical coupling in the samples. Magnetomechanical coupling coefficient $k$ was determined from the resonant $\left(f_{\mathbf{r}}\right)$ and antiresonant $\left(f_{\mathrm{a}}\right)$ frequencies of the free vibrating samples in the form of strips

$$
k=\frac{\pi}{\sqrt{8}} \sqrt{\frac{f_{\mathrm{a}}^{2}-f_{\mathrm{r}}^{2}}{f_{\mathrm{a}}^{2}}} .
$$

The dynamical impedance $Z$ of the measuring resonant circuit was also considered. Magnetic domain structures were studied using Kerr effect method. 


\section{Results and discussion}

The results of the measurements of the magnetomechanical coupling coefficient $(k)$ versus static magnetic field are presented in Fig. 1a. Corresponding curves of dynamical impedance $Z$ after annealing treatments at different temperatures are shown in Fig. 1b. The maximum value of the magnetomechanical coupling coefficient increases with the annealing temperature (up to $510^{\circ} \mathrm{C}$ ), reaching 0.64 . Thus the magnetomechanical coupling of the $\mathrm{Fe}-\mathrm{Si}-\mathrm{B}-\mathrm{Cu}-\mathrm{Nb}$ alloy is several times stronger than in traditional polycrystalline piezomagnetic materials, however it is still about $25 \%$ worse than in the best metallic glasses, e.g. [3, 4]. After annealings at higher temperatures magnetomechanical coupling decreases rapidly. The heat treatment at $580^{\circ} \mathrm{C}$ leads to the magnetomechanical coupling as small as 0.08 , and annealing at $600^{\circ} \mathrm{C}$ makes the coupling undetectable within this method. The decrease in magnetomechanical coupling can be explained by the drop of magnetostriction with the onset of the nanocrystallization [5-7]. The decrease in the maximum dynamical impedance begins only at the temperatures above $550^{\circ} \mathrm{C}$, when the nanocrystalline phase is well developed. This is the result of competition between magnetoelastic and magnetic properties. The signal induced in the measuring coil becomes unchanged or even increases in spite of the decrease in magnetostriction. This is due to simultaneous improvement of magnetic properties such as decrease in magnetic anisotropy and magnetic hysteresis and increase in magnetic permeability. The biasing field corresponding to the position of the maximum of magnetomechanical coupling, as well as saturation field, decrease with the annealing temperature, which is the evidence for improvement of soft

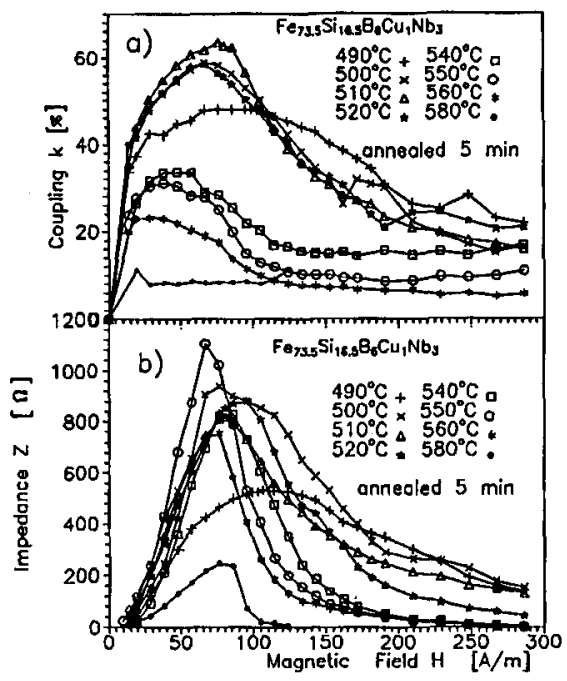

Fig. 1. Magnetomechanical coupling coefficient $k$ (a) and dynamical impedance $Z$ (b) versus magnetic biasing field $H$ for different annealing temperatures $(490,500,510,520$, $540,550,560$, and $\left.580^{\circ} \mathrm{C}\right)$. 
magnetic properties. Magnetomechanical coupling coefficient of multi-domain systems strongly depends on particular domain structure. Suitable domain structures, leading to the best piezomagnetic parameters, can be produced by application of magnetic field during annealings. Characteristic magnetic domain structures for different annealing conditions are illustrated in Fig. 2. The domain structure in Fig. 2a is typical of metallic glasses, in which not all residual stresses are released. The domain pattern in Fig. $2 \mathrm{~b}$ is characteristic of metallic glasses with
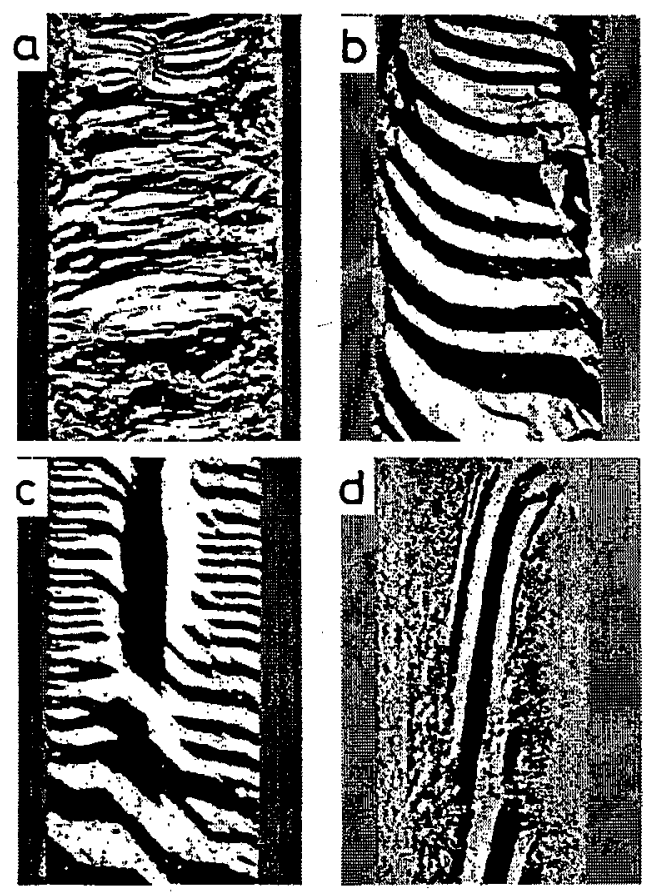

Fig. 2. Typical magnetic domain structures after 5 minute annealings at the following temperatures: (a) $490^{\circ} \mathrm{C}$, (b) $510^{\circ} \mathrm{C}$, (c) $550^{\circ} \mathrm{C}$ and (d) $560^{\circ} \mathrm{C}$.

induced transverse anisotropy and almost free of residual stresses. Samples shown in Figs. $2 c$ and $2 d$ represent the nanocrystalline state. It can be noticed that the volume of domains, which are oriented longitudinally, increases with the annealing temperature. This can be additional reason for the steep decrease in magnetomechanical coupling in the nanocrystalline alloys. Thus, in this case, the decrease in magnetomechanical coupling can be bigger than that expected only from the dependence of magnetostriction on the nanocrystallization degree.

\section{Conclusions}

Iron-rich nanocrystalline materials with small content of nanocrystalline phase exhibit good piezomagnetic properties and their magnetomechanical coupling reaches the value of 0.64 . The value of magnetomechanical coupling coefficient 
as a function of nanocrystalline phase content depends both on magnetostriction and magnetic properties. In spite of improvement of magnetic properties, the drop of magnetostriction and the change of the domain structure cause considerable decrease in magnetomechanical coupling coefficient with the onset of nanocrystallization.

\section{References}

[1] Y. Yoshizawa, S. Oguma, K. Yamauchi, J. Appl. Phys. 64, 6044 (1988).

[2] Z. Kaczkowski, Arch. Elektrotech. (Poland) 11, 635 (1962).

[3] M. van der Brouha, J. van der Borst, J. Appl. Phys. 50, 7594 (1979).

[4] C. Modzelewski, H.T. Savage, L.T. Kabacoff, A.E. Clark, IEEE Trans. Magn. MAG-17, 2837 (1981).

[5] H.R. Hilzinger, J. Magn. Magn. Mater. 83, 370 (1990).

[6] G. Herzer, J. Magn. Magn. Mater. 112, 258 (1992).

[7] Z. Kaczkowski, L. Małkiński, M. Müller, Key Eng. Mater. 81-83, 389 (1993). 